\title{
DIFFUSION ET RÉCEPTION DE LA DYNAMIQUE LA CORRESPONDANCE ENTRE LEIBNIZ ET WOLFF
}

\author{
Anne-Lise ReY
}

RÉSUMÉ: À travers l'analyse de la correspondance entre Leibniz et Wolff, l'article cherche à restituer les modalités démonstratives utilisées par Leibniz pour transmettre sa dynamique ainsi que le dispositif métaphysique qui lui est inhérent. Il est question ici de l'ambivalence du vocable d'action, à la fois objet du principe de conservation et essence de la substance. C'est en étudiant sa réception par Wolff qu'il est possible de mettre en évidence, dès 1705, la singularité d'un cadre métaphysique wolffien qui accueille la dynamique leibnizienne de l'action, puis la publicise, tout en proposant une acception irréductiblement différente de la substance.

Mots-CLÉs: dynamique, ambivalence de l'action, substance, cadre métaphysique.

ABSTRACT: By analysing the correspondence between Leibniz and Wolff, this article aims to present the expository modalities used by Leibniz to transmit his dynamics and the metaphysical system underpinning it. The point is here to study the ambivalence of the vocabulary of action, both as object of the conservation principle and as the essence of substance. By examining the way Wolff receives the dynamics of action, it is possible to highlight, as early as 1705, the uniqueness of his metaphysical framework. While publicizing the dynamics, he propounds a meaning of substance that is unequivocally different.

KEYWORDS : dynamics, ambivalence of action, substance, metaphysical frame. 
ZuSAMMENFASSUNG: Ausgehend von einer Untersuchung der Korrespondenz zwischen Leibniz und Wolff versucht der Artikel, die demonstrativen Modalitäten aufzuzeigen, die Leibniz verwendet hat, um seine Dynamik und das ihr inhärente metaphysische Dispositiv zu vermitteln. Es geht hier um die Ambivalenz der Vokabel "Wirkung» als Gegenstand eines Erhaltungsprinzips und zugleich Essenz der Substanz. Durch eine Untersuchung der Rezeption von Wolff ist es möglich, ab 1705 die Einzigartigkeit eines Wolffschen metaphysischen Rahmens herauszuarbeiten, der die Leibnizsche Dynamik der "Wirkung» aufgreift, publik macht, und dabei eine unreduzierbar von der Substanz verschiedene Bedeutung vorschlägt.

STICHWÖRTER: Dynamik, Ambivalenz der «Wirkung», Substanz, metaphysischer Rahmen.

$$
\text { تقبل العلم الديناميكي و شيو عه. تر اسل ليينتس و فولف، تأليف آن ليز ري. }
$$

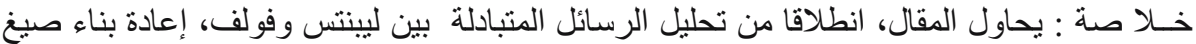

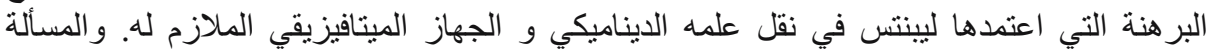

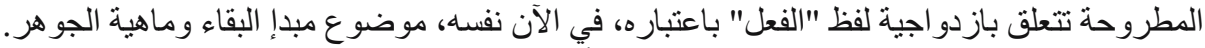

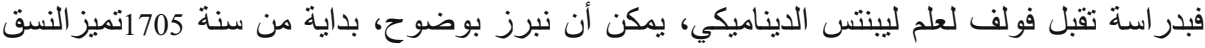

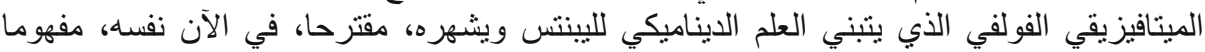

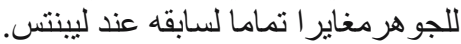

الكلمات المفاتيح : العلم الديناميكي، إزدو اجية الفعل، الجوهر، النسق الميتافيزيقي. 
La dynamique de Leibniz, au sens strict d'une dynamique de la puissance et de l'action, telle qu'elle est élaborée à partir du texte de 1689-1690, la Dynamica de potentia $^{1}$, développe une relation de fécondation réciproque entre un principe de conservation de la quantité d'action, qui s'estime selon les forces et les temps, et la définition de la substance entendue comme essentiellement active. C'est une science mixte en ce qu'elle lie étroitement la physique et la métaphysique, comme Leibniz l'indique par exemple en 1694 dans De la réforme de la philosophie première lorsqu'il écrit: «[...] la notion de force à laquelle j'ai consacré une science spéciale, la Dynamique, apporte beaucoup de lumière à l'intelligence de la vraie notion de substance ${ }^{2}$.»

Par ailleurs, comme l'a montré Michel Fichant dans son article «De la puissance à l'action: la singularité stylistique de la Dynamique ${ }^{3} »$, les modalités de la diffusion de la pensée de Leibniz font que cette relation réciproque entre la définition de la substance par l'action et le principe de conservation de l'action ${ }^{4}$, même si elle est évoquée dans certains textes publiés à la fin des années 1690 dans les journaux savants, n'a jamais été véritablement fondée ailleurs que dans des correspondances ${ }^{5}$ avec quelques interlocuteurs choisis que furent Johann Bernoulli, Denis Papin, Burcher De Volder et Christian Wolff.

Notre projet est alors de lire cet échange entre Leibniz et Wolff comme un laboratoire qui permet de voir, au fur et à mesure que Leibniz dévoile sa théorie et ses implications, la part de leibnizianisme à laquelle Wolff adhère et celle à laquelle il s'oppose. Nous voudrions tout d'abord restituer les éléments - délivrés par Leibniz à Wolff dans le cadre de leur correspondance - qui permettent de comprendre selon quelle logique Leibniz a diffusé auprès de Wolff cette intrication entre action motrice et essence de la substance. Cette intrication nous semble être le cœur de l'enjeu et de la fécondité de la dynamique leibnizienne. On procèdera donc au repérage des arguments fournis par Leibniz à Wolff afin de voir comment Wolff s'y oppose, y répond ou y adhère.

Les textes significatifs de Wolff pour cette question se trouvent tout d'abord dans les Principia dynamica publiés en 1728 dans les Mémoires de l'académie de St-Pétersbourg ${ }^{6}$, et dans la Cosmologia generalis, au chapitre IV de la section II consacré aux lois du mouvement ${ }^{7}$. On y trouve une fidélité assez scrupuleuse à l'égard de l'estimation du principe de conservation de l'action à l'œuvre dans la dynamique de Leibniz. Mais à lire certains paragraphes de l'Ontologia $^{8}$ et d'autres paragraphes

1. LEIBNIZ, 1689-1690.

2. LeIBNIZ, 1694, ici 1978 , p. 81.

3. Voir Fichant, 1998, p. 220 et suiv.

4. Leibniz, 1698, ici 1978, p. 101: «Pour ma part, si, comme je le crois, j’ai bien compris la notion d'action, j'estime qu'elle implique et justifie le principe philosophique unanimement reçu, que toute action est l'action d'un sujet individuel. Et je trouve ce principe si vrai que sa réciproque l'est aussi, c'est-à-dire que non seulement tout ce qui agit est une substance individuelle, mais aussi que toute substance individuelle agit sans interruption, et je n'en excepte même pas le corps, car on n'y remarque jamais de repos absolu.»

5. Voir Phemister, 2005, chap. I, p. 1-29.

6. WoLfF, 1728.

7. WoLfF, 1731, ici 1964, p. 228-392.

8. WoLfF, 1730. Pour le principe de raison suffisante, voir part. I, sec. I, chap. II, §56-78, par exemple le scholie du $\S 75$, et surtout pour la définition de la substance, part. II, sect. II, chap. I-II. 
de la Cosmologia generalis, on observe, comme l'a relevé Jean École dans son texte «Cosmologie wolffienne et dynamique leibnizienne ${ }^{9} »$, une identité plus apparente que réelle dans la mesure où l'on peut lire sous la plume de Christian Wolff la formulation de deux divergences fondamentales qui l'opposent à Leibniz. Il s'agit à la fois d'une divergence métaphysique qui porte sur la définition de la notion de substance et d'une divergence que l'on pourrait caractériser d'architectonique dans la mesure où elle porte sur le rapport entre la physique et la métaphysique, tel qu'il s'exprime de manière si singulière dans la dynamique leibnizienne.

Il y a ainsi, chez Wolff, un refus d'associer force primitive et entéléchie (par exemple dans le $\S 361$ de la Cosmologia generalis ${ }^{10}$ ). Il y a également un refus d'identifier ses éléments à la monade (toujours dans la Cosmologia generalis dans la note du $\S 182^{11}$ ). Les atomes de substance ou points métaphysiques sont en effet définis par Leibniz dans le Système nouveau de la nature et de la communication des substances de 1695 comme ayant «quelque chose de vital et une espèce de perception ${ }^{12}$ », alors que pour Wolff les éléments simples sont des «points physiques indivisibles ${ }^{13} »$. Or, pour Leibniz, l'entéléchie et la perception sont les éléments clefs de l'action pensée comme essence de la substance. Ce que récuse donc Wolff c'est le fondement métaphysique de la dynamique par l'action. Par ailleurs, on relèvera qu'à rebours Wolff ne prend pas non plus acte, ni en amont, ni en aval de la dynamique, des impacts de la découverte du principe de conservation de l'action pour définir la substance. C'est ce qui explique que sa substance ou son élément simple soit non perceptif et physique.

L'un des enjeux de l'étude de cette correspondance sera donc de comprendre pourquoi on trouve chez Wolff ce paradoxe d'une grande fidélité à l'égard de la diffusion de la dynamique entendue dans sa partie mathématique (c'est-à-dire de son principe de conservation de la même quantité d'action et des modalités démonstratives a priori qui l'accompagnent) et cette infidélité à l'égard de la fécondité métaphysique de la dynamique. Comprendre ce paradoxe c'est mieux comprendre, pensons-nous, le cadre métaphysique propre à la pensée de Wolff.

Comment dès lors est-il possible de diffuser la dynamique leibnizienne (dans les Principia dynamica par exemple) sans ce qui est à son origine et comme sa conséquence, à savoir ce qui engage la définition de la substance chez Leibniz? Autrement dit, sur quoi Wolff fonde-t-il l'estimation de la dynamique de l'action, si ce n'est sur ce qui la fonde chez Leibniz? À cet égard, il pourrait être opportun d'interroger la place que prend la pensée de Newton chez Wolff ${ }^{14}$. Ces questions qui intéressent la diffusion, pensée comme traduction, se déploient dans un cadre métaphysique qui est propre à Wolff et qu'on voit se dessiner dès la correspondance: si elle est bien ce laboratoire du

9. ÉCOLE, 1964.

10. WoLfF, 1731, ici 1964, p. 261-262.

11. WoLfF, 1731, ici 1964, p. 146: «[...] Sed nos elementorum appellatione contenti monadum nomine non utemur.»

12. LeIBNIZ, 1695, ici 1994, p. 71.

13. WolfF, 1731, ici 1964, p. 166: «les atomes de nature sont des points physiques ».

14. Voir Anne-Lise ReY, « Liebniz et Newton dans Wolff: un précurseur pour les Lumières européennes? », article à paraître dans le recueil La Pensée encyclopédique de Christian Wolff. Autour du Discursus praeliminaris (Paris, Vrin). 
«leibnizianisme» de Wolff, sa lecture nous rend témoins de la constitution et parfois de la formulation des différences de positions. Nous présenterons donc les étapes par lesquelles Leibniz procède au dévoilement de ses arguments concernant l'ambivalence de l'action à l'œuvre dans la dynamique. Puis, nous interrogerons la fidélité de Wolff vis-à-vis de la dynamique.

\section{LES ÉTAPES D’UN DÉVOILEMENT}

Par quelles étapes passe le dévoilement des arguments leibniziens relatifs à l'ambivalence de l'action? Le point de départ de ce travail est de mesurer, par le biais de l'analyse de la correspondance avec Leibniz, si Wolff a fait partie des quelques heureux correspondants auxquels Leibniz a confié le rapport étroit et réciproque, en un mot fondationnel, qu'il introduisait entre principe de conservation de l'action et resémantisation de la substance et qui a une place centrale dans ce que Fichant a désigné comme «l'invention métaphysique ${ }^{15}$ » propre à Leibniz. Si c'est le cas de quelle manière cela a-t-il été transmis, et comment Wolff l'a-t-il reçu? On peut rappeler schématiquement que pour Leibniz, au cœur de la dynamique de l'action se trouve une action formelle ou action sur soi qui fonde l'action violente. Or, cette action sur soi, métaphysique, fonde la dynamique, comme en atteste la présence d'un vocable métaphysique situé au cœur de la dynamique. À rebours, cette action dynamique permet de redéfinir la substance comme ayant pour essence même l'action sur soi.

\section{La transmission d'un dispositif métaphysique}

Leibniz transmet d'abord à Wolff un dispositif métaphysique assez complet dans lequel il donne les éléments nécessaires à la compréhension du rapport qu'il établit entre substance simple et substance corporelle. À cet égard, il se cantonne, délibérément, au cadre du rapport entre l'âme et le corps, celui-ci étant conçu comme une sorte de modèle pour comprendre et expliquer ce rapport. Ainsi, Leibniz ne procède pas comme dans la correspondance avec De Volder, où il diffusait également la démonstration a priori du principe de conservation de l'action et où il explicitait les relations de fécondation réciproque de l'action métaphysique et de l'action dynamique. Dans la correspondance avec De Volder, en effet, Leibniz abordait directement la question du rapport entre substance simple et substance corporelle, car il avait montré dans la première partie de sa correspondance l'incomplétude réciproque de la dynamique et de la métaphysique. Il avait, à partir de là, introduit la notion d'entéléchie comme un moyen de surmonter cette incomplétude, corrélativement il pouvait mettre en évidence l'ambivalence de l'action. On voit ainsi, comment les procédures démonstratives diffèrent selon les problèmes qui nouent les correspondances puisque dans la lettre 14, adressée à Wolff, Leibniz indique que «dans les choses externes, de même que dans l'âme, il y a deux choses: l'état et la tendance à un autre état ${ }^{16} \gg$. Il fait ainsi de l'âme un modèle pour penser sans distinction majeure les choses externes et les éléments internes et pour affirmer la présence en toute forme de réalité de deux éléments, garants en quelque sorte de leur intelligibilité. Il affirmait,

15. FichANT, 2004.

16. Lettre de Leibniz à Wolff, non datée, dans Leibniz, 1705-1716, ici 1971, lettre n 14, p. 56. 
déjà dans la lettre du 9 novembre 1705, «d'après moi, selon la grande uniformité de la nature, toutes choses partout dans les grandes et dans les petites, dans les visibles et dans les invisibles se produisent de la même manière, et varient seulement par leur degré de grandeur et de perfection ${ }^{17} \gg$.

On peut retenir de ce point l'affirmation leibnizienne selon laquelle il n'y a pas de différences de nature entre les éléments dotés de réalité. Il peut donc, à ce titre, mobiliser le rapport entre l'âme et le corps comme un «modèle d'intelligibilité» sans qu'il soit pour autant légitime de considérer sa théorie comme un spiritualisme ou un panpsychisme. À partir de la restitution de ce cadre explicatif, Leibniz explicite un premier élément au fondement de sa définition de la substance, telle qu'elle a été reformulée grâce aux apports de la dynamique: la substance est perceptive, dire qu'elle est comme une âme ne signifie pas nécessairement qu'elle est spirituelle, mais simplement qu'elle est douée d'une activité qui la rend, comme il le dira à la fin de la correspondance, «monadique et dynamique». Il explicite donc la place centrale de la perception en rappelant qu'en toute âme, il y a à la fois l'expression d'un état des choses extérieures ${ }^{18}$ et la tendance à une nouvelle expression, qu'il appelle perception et perceptibilité. Il fait ensuite une distinction entre âme et esprit qui lui permet de récuser la réduction cartésienne à la conscience, qui limite la perception aux esprits ${ }^{19}$.

\section{La réception wolffienne du dispositif métaphysique leibnizien}

Comment Wolff reçoit-il cette conception métaphysique qui correspond à peu près à ce qu'il a pu lire dans les textes que Leibniz a fait paraître dans les journaux savants quelques années auparavant? L'idée que la substance soit par essence perceptive, ainsi que la nécessité de distinguer l'âme de l'esprit, et que cette âme, entendue comme quelque chose de substantiel soit identifiée à la monade se trouve en effet dans le De ipsa natura ${ }^{20}$. La particularité de la pensée de Leibniz sur ce point, comme on l'a dit, est qu'il ne fait pas de différence de nature entre les formes de réalité. Or, dans le cadre de sa correspondance avec Leibniz, Wolff ne réagit pas directement à cette introduction de la perception et de la perceptibilité. Plus tard, dans la Psychologia rationalis ${ }^{21}$ il récusera l'idée qu'une perception soit logée dans toutes les formes de substance, en distinguant bien, comme l'a montré Yvon Belaval dans ses Études leibniziennes $^{22}$, les esprits qui conservent le nom de monades et auxquels Wolff attribue la perception et les monades brutes auxquelles Wolff refuse la perception et qu'il désigne comme des atomes de nature.

La lettre ${ }^{23}$ qui suit immédiatement celle dans laquelle Leibniz rappelle sa position sur la perception présente en toute forme de réalité (et non seulement dans les

17. Lettre de Leibniz à Wolff du 9 novembre 1705, dans LeIBNIZ, 1705-1716, ici 1971, lettre n 8, p. 44.

18. Lettre de Leibniz à Wolff, non datée, dans LeIBnIz, 1705-1716, ici 1971, lettre ${ }^{\circ} 14$, p. 56.

19. Lettre de Leibniz à Wolff, non datée, dans Leibniz, 1705-1716, ici 1971, lettre $n^{\circ} 14$, p. 56.

20. LeIBNIZ, 1698, ici 1978, p. 105.

21. WoLfF, 1734, voir par exemple les $\S 644$ et 712 .

22. Voir Belaval, 1976, p.160 et suiv., au chapitre consacré à la perception.

23. Il s'agit de la lettre de Wolff à Leibniz du $1^{\text {er }}$ octobre 1708, dans LeIBNIZ, 1705-1716, ici 1971, lettre $\mathrm{n}^{\circ} 37$, p. 98 . 
substances spirituelles) est celle dans laquelle Wolff formule l'un des problèmes le plus longuement discuté dans la correspondance: celui du statut des forces dérivatives et de leur rapport aux forces primitives. On peut se demander quel rapport existe entre la formulation leibnizienne et la question wolffienne qui lui succède et en quelque sorte lui répond: l'enjeu pour Leibniz est justement de penser la relation entre la force primitive et la force dérivative, sur le mode d'une modification de l'une pour produire l'autre. Toute la démarche de Wolff sera de les penser comme distinctes. À travers cette séparation, il s'agit bien, pour Wolff, de poser une différence irréductible entre le niveau de phénoménalité propre aux forces dérivatives et celui, proprement substantiel, dont relève la force primitive.

Wolff comprend au début de l'échange, la force dérivative comme «ce qui a le pouvoir de se transporter d'un corps à un autre ${ }^{24} \gg$ et il en tire comme conséquence le nécessaire recours à l'action immédiate de Dieu, convoquée par les cartésiens. Leibniz y répond dans la lettre suivante en substituant à la causalité directe proposée par Wolff, l'idée d'une causalité idéale fondée sur l'hypothèse de l'harmonie préétablie et qui suppose de penser, de manière spécifique, le rapport entre force primitive et force dérivative ${ }^{25}$.

C'est sur l'explication de la relation entre force primitive et force dérivative que va porter une partie des échanges à venir. Leibniz la fonde sur l'hypothèse de l'harmonie préétablie et cette explication - qui suppose de lire les forces dérivatives comme des modifications de quelque chose qui persiste et qui est substantiel, à savoir les forces primitives - lui permet d'introduire conjointement la notion d'entéléchie, assimilée à un actif substantiel et la justification du recours à la dynamique. La démonstration de Leibniz procède en trois temps. Il indique tout d'abord dans une lettre de novembre 1710 que «la raison des modifications de la force primitive est la même que la raison des mouvements ${ }^{26} »$, et il précise qu'elle n'est pas intelligible à partir des pures mathématiques. Il amorce par là l'introduction de la dynamique. Puis, dans une lettre du début de l'année 1711, il rappelle le clivage entre l'activité de la force primitive et sa modification, passive, dans la force dérivative en rappelant que la modification doit être lue comme une détermination: «On peut dire qu'il y a une force en tout corps et n'est déterminée que par la seule modification ${ }^{27}$.» Enfin, dans la lettre du 9 juillet 1711, il explicite l'analogie entre force primitive et monade d'un côté et force dérivative et phénomène, de l'autre, de sorte que:

«[...] la modification de la force primitive, qui est dans une Monade, ne peut pas être mieux expliquée qu'en exposant comment la force dérivative change dans les

24. Lettre de Wolff à Leibniz du $1^{\text {er }}$ octobre 1708 , dans LeIBNIZ, 1705-1716, ici 1971 , lettre ${ }^{\circ} 37$, p. 98.

25. Lettre de Leibniz à Wolff, non datée, dans Leibniz, 1705-1716, ici 1971, lettre ${ }^{\circ}$ 38, p. 99 : «Je n'ai jamais dit que la force dérivative se transférait d'un corps dans un autre, mais que la suivante naissait dans chaque substance de la précédente à l'occasion d'une autre substance et pour ainsi dire par une conspiration [accord].»

26. Lettre de Leibniz à Wolff, non datée, dans LeiBniz, 1705-1716, ici 1971, lettre n 59, p. 129.

27. Lettre de Leibniz à Wolff, dans Leibniz, 1705-1716, ici 1971, lettre n 61, p. 131 (voir également l'ensemble des pages 130 et 131). La lettre n'est pas datée, mais elle se situe entre celle du 31 décembre 1710 et celle du 16 avril 1711. 
phénomènes. Car ce qui, dans les phénomènes, est montré de manière étendue et mécaniquement, dans les Monades est concentré et doté d'un principe de $v^{28}{ }^{28}$.»

Leibniz l'articule ensuite à la question du mode comme variation de l'essence, entendue comme possibilité de la chose, en disant de la même manière que les modifications de la monade naissent d'elle-même, comme des modes de son essence et non d'une autre chose. La position de Wolff, même si elle n'est pas explicitement exprimée, peut s'entendre à partir des questions que l'exposé leibnizien conduit à soulever. On peut pointer deux types de questionnement. Ainsi, Wolff assimile en premier lieu la force primitive et l'essence de la matière. Cela lui permet de maintenir l'idée d'un transfert de forces d'un corps à un autre, donc l'idée d'une causalité directe. On retrouvera cette idée dans une perspective un peu différente dans la Cosmologia generalis aux $\S 299$ et 300 où pour Wolff, c'est l'état présent qui est la cause de l'état suivant d'un élément. L'enjeu est bien l'explication de l'accord - ou conspiration - à l'origine de l'hypothèse de l'harmonie préétablie. Or, au début de la correspondance, il semble encore apprécier l'hypothèse de l'harmonie préétablie et il l'indique. Dans la lettre du 2 décembre 1705, il écrit:

«Le système de l'Harmonie préétablie me plaît prodigieusement, d'abord parce qu'il est plus digne d'un philosophe que l'appel à la volonté immédiate de la puissance divine et surtout parce qu'il fait beaucoup pour promouvoir la Sagesse de Dieu ${ }^{29}$.»

Il n'est pas sûr, y compris dès cette date, que cette admiration résiste aux questions posées sur les rapports entre force primitive et force dérivative. La réponse de Leibniz consistera à rappeler la différence entre matière et corps qu'il avait posée dans le De ipsa natura grâce à la différence entre matière première et matière seconde ${ }^{30}$.

En second lieu, la question est précisée dans la lettre du 26 juin 1711, c'est-à-dire après le dévoilement de la démonstration a priori des lois de la dynamique ${ }^{31}$. Wolff demande comment Leibniz explique la modification de la force primitive, par exemple quand le mouvement s'accélère dans le grave descendant. Et c'est à partir de cette question que Wolff assimile la force primitive à la force d'inertie. Il reprend ainsi, l'acception newtonienne de la «vis insita» entendue comme force d'inertie présente dans les Principes mathématiques de la philosophie naturelle ${ }^{32}$. Ce qui est en jeu, c'est la

28. Lettre de Leibniz à Wolff du 9 juillet 1711, dans LeIBNIz, 1705-1716, ici 1971, lettre $n^{\circ}$ 67, voir en part. p. 138-139 (voir également l'ensemble des pages 138 et 139).

29. Lettre de Wolff à Leibniz du 2 décembre 1705, dans Leibniz, 1705-1716, ici 1971, lettre n 9, p. 46.

30. LeIBNIz, 1698, ici 1978, p. 105: «[...] il faut distinguer la matière seconde et la matière première, la seconde est une substance complète, mais n'est pas purement passive, la première est purement passive, mais n'est pas une substance complète. Il doit donc s'ajouter à cette dernière une âme ou une forme analogue à l'âme, ou une entéléchie première, c'est-à-dire une certaine tendance ou force primitive d'agir qui est une loi inhérente à cette substance et lui a été imprimée par un décret de Dieu.»

31. Lettre de Wolff à Leibniz du 26 juin 1711, dans LeibnIz, 1705-1716, ici 1971, lettre nº 64, p. 136.

32. Newton, 1687, ici 1985, p. 25, liv. I, déf. 3 : «La vis insita d'une matière est la force de résistance par laquelle tout corps, autant qu'il le peut, persévère en son état de repos ou de mouvement rectiligne uniforme.» 
définition même de la force primitive que Wolff conçoit comme ce dont la modification produit l'impetus; la force d'inertie est la force par laquelle un corps résiste à l'impetus d'un autre corps. Par là, il l'identifie à une force passive ou «force d'inactivité » dira Max Jammer ${ }^{33}$ dans son ouvrage Concepts of force, alors que pour Leibniz dans ce contexte explicatif, la notion de force primitive est clairement comprise comme celle de force active primitive. De sorte que la différence qui s'esquisse ici porte sur l'assimilation ou non de l'essence de la substance à la force active, à la perception et à ce qui permet de l'expliquer dans la substance, c'est-à-dire à l'entéléchie.

Wolff récuse à la fois l'idée que la perception soit présente au cœur de la substance et l'idée que l'âme soit un modèle d'intelligibilité permettant de décrire, de la même manière, l'activité de toutes les substances. Or, ces deux idées résultent, dans la logique de développement de la pensée de Leibniz, de la fécondité de la dynamique pour la redéfinition de la substance. Wolff pose donc, dès la correspondance, les prémisses d'une séparation, qui prend la forme d'une nette distinction, entre les monades spirituelles dotées de perception et les monades brutes, dépourvues de cette perception. Mais le fait de poser cette séparation induit logiquement le refus de l'argument que Leibniz mobilise dans l'ensemble de leur échange : l'idée qu'il est possible de penser de façon analogique le changement phénoménal et l'activité substantielle en considérant que le premier exprime plus explicitement le second. Toutefois Leibniz ne se contente pas de poser la relation entre les deux sur un mode analogique, puisqu'il précise dans la lettre du 9 juillet 1711 que «les phénomènes résultent des monades qui seules sont les vraies substances ${ }^{34} \gg$. A contrario, Wolff semble maintenir l'idée que la modification de la force dérivative s'explique par ce qu'il appellera plus tard un influx physique, ne prenant pas en considération la relation que Leibniz introduit avec la notion d'entéléchie pour fonder l'idée d'un accord idéal. Comment, dès lors, Wolff accueille-t-il la dynamique au sein d'un cadre conceptuel métaphysique distinct du cadre leibnizien?

\section{La fonction de la dynamique dans les échanges entre Leibniz et Wolff}

Leibniz introduit le terme de «dynamique», pour la première fois dans la lettre du 20 août $1705^{35}$. Il y est conduit par la nécessité d'introduire la notion de substance corporelle qui «enveloppe une chose dynamique», dans la mesure où le corps ne permet pas par lui-même de rendre raison des lois du mouvement. Puis, Leibniz revient sur la dynamique sans la mentionner explicitement en rappelant la confusion cartésienne entre quantité de mouvement et quantité de force, et en rappelant la différence entre son explication du mouvement des corps et celle des cartésiens qui recourent à la création continuée de Dieu ou, pour certains d'entre eux, à l'occasionnalisme. La dynamique est ainsi présentée par Leibniz comme un moyen d'expliquer, par le biais de la substance corporelle, les lois du mouvement. Elle permet, contre Descartes, d'établir

33. JAMMER, 1957, ici 1999, p. 119, cité par FiCHANT, 1998, p. 241 : «The inert nature of mater is here conceived as a force of inactivity. "

34. Lettre de Leibniz à Wolff du 9 juillet 1711, dans LeIBNIZ, 1705-1716, ici 1971, lettre $\mathrm{n}^{\circ} 67$, p. 139

35. Lettre de Leibniz à Wolff du 20 août 1705, dans LeIBNIZ, 1705-1716, ici 1971, lettre n 6, p. 34. 
le véritable principe de conservation et elle est une explication qui ne suppose pas le recours à la création continuée cartésienne.

La teneur de la réception wolffienne de ce propos, présente dans la lettre du 6 novembre $1708^{36}$, touche au cœur de la dynamique. S'appuyant sur une citation de Parent et de ses Disquisitiones mathematicae, il pose la question suivante: comment faire de l'action sur soi une action qui ne résulte pas d'une machine et qui ne porte pas atteinte à la toute-puissance divine? C'est la question de la justification de la notion d'automate spirituel qui est posée, et de la manière d'expliquer la raison de cette action sans recourir à l'action immédiate de Dieu.

La réponse de Leibniz ${ }^{37}$ consiste à réaffirmer la relation étroite entre l'hypothèse de l'accord présente dans la théorie de l'harmonie préétablie (qui suppose certes le concours de Dieu, mais pas son concours miraculeux) et la notion d'action sur soi, car loin de pouvoir être assimilée à une machine, elle est la racine de l'expression du préordonnancement divin. Il semblerait donc que Wolff, maintenant l'idée d'une causalité physique et non idéale, soit conduit, dès sa correspondance avec Leibniz, à renoncer à l'hypothèse de la conspiration, présente dans la théorie de l'harmonie préétablie, même s'il ne le dit pas explicitement. Puis, à la demande faite par Wolff de savoir ce qu'est le principe universel à partir duquel Leibniz démontre les lois du mouvement ${ }^{38}$, ce dernier formule sa réponse sur les principes de la mécanique en recourant à l'axiome métaphysique de l'équivalence de la cause pleine et de l'effet entier, mais en précisant qu'il a son fondement ultime dans la sagesse divine ${ }^{39}$.

À partir de là, il paraît logique que Wolff propose une démonstration de l'estime leibnizienne des forces vives basée sur l'axiome métaphysique de la cause pleine et de l'effet entier. Mais dans cette démonstration, Leibniz lui reproche trois choses ${ }^{40}$ : 1) de ne pas avoir explicité que par mouvement, il entendait en fait action motrice; 2) de faire comme ceux qui considèrent la quantité de mouvement en proportion de la vitesse et non du temps, s'il en fait l'estime par le degré de vitesse; 3) de considérer que l'impetus ne doit pas être mesuré par le temps, mais par l'effet, c'est-à-dire l'espace parcouru. Leibniz doute qu'il soit pertinent d'attribuer l'impetus à l'espace. Cela ne peut venir de l'axiome d'équivalence entre la cause et l'effet car dans cet axiome, les effets sont compris comme absorbant la cause et la vitesse n'est pas prise en considération. En revanche, dans les actions pures, les actions sont en raison composée des effets et des vitesses. Il en délivre une démonstration dont il rappelle qu'il l'a commu-

36. Lettre de Wolff à Leibniz du 6 novembre 1708, dans LeIBNIZ, 1705-1716, ici 1971, lettre nº 39, en part. p. 100.

37. L'essentiel du propos de Parent - tel qu'il est cité par Wolff dans sa lettre du 6 novembre 1708, dans LeIBNIZ, 1705-1716, ici 1971, p. 100-101 - revient premièrement à évaluer les thèses de Leibniz à l'aune des thèses cartésiennes: "Au contraire Leibniz dit moins bien que les Cartésiens », car tout en affirmant la prescience de Dieu, Leibniz n'explique les changements dans les corps ni par le recours à l'action immédiate de Dieu, ni par la création continuée. Il revient deuxièmement à pointer les conséquences de la doctrine leibnizienne: une âme semblable à une machine et n'ayant pas besoin de l'action immédiate de Dieu puisque agissant par elle-même. «Ce qu'on ne peut catholiquement penser.»

38. Lettre de Wolff à Leibniz du 6 novembre 1708, dans LeiBnIz, 1705-1716, ici 1971, lettre n 39, p. 102.

39. Lettre de Leibniz à Wolff, dans LeiBnIz, 1705-1716, ici 1971, lettre ${ }^{\circ} 59$, p. 129. Cette lettre n'est pas datée mais se situe entre celle du 8 novembre 1710 et celle du 31 décembre 1710 .

40. Lettre de Leibniz à Wolff, non datée, dans LeibNIz, 1705-1716, ici 1971, lettre n 61, p. 131. 
niquée il y a longtemps à Johann Bernoulli. Il la désigne comme métaphysique, c'està-dire «abstraite de la gravité, de l'élasticité de la composition des choses physiques et des suivantes ${ }^{41} »$.

L'ensemble de ces traits distinctifs dessine le cadre métaphysique de la pensée de Wolff depuis lequel il va diffuser la dynamique de Leibniz, mais cela dessine également le sens que Wolff attribue à l'usage de la métaphysique pour fonder la dynamique: une métaphysique des principes.

\section{QUELLE FIDÉLITÉ À LA DÉMONSTRATION DES LOIS DE LA DYNAMIQUE?}

Il s'agit ici de comparer la démonstration donnée par Leibniz à Wolff dans leur correspondance avec celle donnée par Wolff dans les Principia dynamica ${ }^{42}$. Un glissement s'opère: en effet, Wolff fonde sa démonstration sur des principes métaphysiques et non sur des concepts ${ }^{43}$, autrement dit Wolff conserve l'idée d'une relation étroite entre la dynamique et la métaphysique, mais au lieu, comme le fait Leibniz, de donner une place à la notion ambivalente d'action ${ }^{44}$, comme un concept mixte, il fonde la dynamique sur un certain nombre de principes métaphysiques.

Mentionnons rapidement la forme que prend la transmission de la démonstration a priori des lois de conservation de la même quantité d'action motrice, qui conduit à établir que l'action s'estime selon les temps et les puissances. Leibniz reprend le cheminement de la démonstration déjà produite pour Johann Bernoulli et De Volder, en remplaçant le motif du double par celui de la dimidiation ${ }^{45}$, avec deux modes de formulation: celui qui figure un schéma et celui qui procède par lettres; et il rend compte de deux cas (le mouvement égal et le mouvement inégal). Leibniz ne parle pas, ici, d'action formelle, mais d'action pure. Même s'il mobilise la double résolution de l'action, soit en fonction des temps et des puissances soit en fonction des effets ou des espaces et des vitesses, il ne la réfère pas à la double résolution selon l'intension et l'extension comme il le faisait pour De Volder et pour Johann Bernoulli. Cette double résolution rapproche l'estime de l'action du motif médiéval des latitudines. Il y a dans le texte des Principia dynamica un certain nombre d'éléments qui indiquent une restitution très fidèle de la dynamique de Leibniz. On en isole trois principaux.

41. Lettre de Leibniz à Wolff, non datée, dans LeIBnIz, 1705-1716, ici 1971, lettre n 61, p. 132.

42. WolfF, 1728 , voir tout particulièrement le $\S 58$, scholie du corollaire 3 de la démonstration du théorème 14.

43. Voir Duchesneau, 1994, p. 331 : «En produisant ses Principia dynamica (1728), Wolff ne se fera pas faute de reconstruire la science leibnizienne comme une déduction a principiis. Ce faisant, il rigidifie sans doute un mode de construction analytique que Leibniz concevait plutôt sur le mode d'une invention combinatoire. »)

44. Nous avons développé l'analyse de cette information réciproque de la dynamique et de la métaphysique opérée grâce au vocable ambivalent d'action dans notre thèse de doctorat soutenue en décembre 2003 sous la direction de Michel Fichant, L'Ambivalence de la notion d'action. Un exemple de diffusion de la dynamique de Leibniz: la correspondance entre Leibniz et De Volder. Une partie de la thèse est en cours de publication.

45. La dimidiation est l'opération qui consiste à diviser en parties égales. 
En premier lieu, il convient de rappeler que la référence à Galilée est largement présente dans les textes consacrés à la dynamique. Dans la préface de la Dynamica de potentia ${ }^{46}$, Leibniz indique clairement son inscription dans une filiation galiléenne. Il place, en effet, son œuvre sous l'égide de Galilée, «louant les monuments immortels de Galilée», puis il indique qu'il se veut le continuateur de l'œuvre de ce dernier, puisqu'à propos de la dynamique, il écrit qu'il s'agira d'une «œuvre qui poursuit ce que Galilée avait commencé». On retrouve également cette référence à Galilée dans l'Essay de dynamique de $1692^{47}$. Elle y apparaît comme fondement de l'ensemble de la démonstration. Il s'agit de la proposition I avec comme sous-titre: «Lemme démontré par les autres ${ }^{48}$.» Leibniz écrit alors : «les vitesses que les corps pesants acquièrent en descendant sont comme les carrés des hauteurs dont ils descendent [...]», et dans le scholie il ajoute que «cette proposition a été démontrée par Galilée, Huygens et les autres». Or, on retrouve la même chose dans les Principia dynamica de Wolff dans le corollaire 2 du théorème 15 qui s'appuie sur la référence à la même loi de Galilée.

En second lieu, la définition leibnizienne de l'action comme l'exercice de la force en des temps égaux se retrouve à l'identique dans le $\S 3$ de l'axiome 2 des Principia dynamica. Enfin, et peut-être même surtout, est présente sous la plume de Wolff, l'idée que l'effet est une détermination métaphysique au cœur de la dynamique et que cette détermination métaphysique, et abstraite, est ce qui permet d'éviter les erreurs. Il confère donc bien à l'action sa détermination idéale comme fondement de l'action violente ${ }^{49}$. Wolff l'indique, par exemple, dans le scholie de la définition 6 où il évoque le refus opéré par Papin dans son texte des Acta eruditorum de 1691 d'accorder l'existence d'une action formelle: «Papin refuse de donner un quelconque effet à la force motrice, quand aucune résistance ne se manifeste ${ }^{50}$.» Or, la définition 6 est la définition de l'effet formel («L'effet de la force motrice hors du choc est le transfert du mobile à travers l'espace») telle qu'on peut la retrouver dans l'Essay de dynamique de 1699-1700: "Cet effet formel ou essentiel au mouvement consiste dans ce qui est changé par le mouvement, c'est-à-dire dans la quantité de masse qui est transférée et dans l'espace ou dans la longueur par laquelle cette masse est transférée ${ }^{51}$.» On retrouve donc, formulée dans les mêmes termes, la même définition de l'effet formel. Ainsi, le moyen pour Wolff de comprendre l'existence de l'action formelle (et donc de récuser la position de Papin) est de prendre en compte la dimension métaphysique de l'effet, dans le scholie de la définition 6: «À partir de la définition de l'effet en général, qui est métaphysique, on réfute l'erreur [de Papin].»

La fidélité à la doctrine dynamique que l'on vient de rappeler est d'ailleurs clairement revendiquée par Wolff qui cite une lettre de Leibniz de 1711, transmise à Johann Bernoulli et à Jacob Hermann, qui a été publiée dans les Acta eruditorum, pour montrer

46. LeIBNIZ, 1689-1690.

47. Costabel, 1981.

48. Costabel, 1981, p. 100.

49. Or, cette idée d'une action formelle conçue comme fondement de l'action violente est au coeur de la dynamique de l'action.

50. WolfF, 1728, ici 1974, p. 154.

51. LeIBNIZ, 1699-1701, ici 1971, p. 220. 
la conformité de son texte à l'esprit de la dynamique leibnizienne. À cet égard, il écrit dans le scholie du corollaire 3 de la définition 6, §58 des Principia dynamica: «C'est pourquoi je ne doute nullement de ce que les principes de la dynamique que je propose ici soient conformes à la pensée de Leibniz, principes par lesquels une voie est ouverte qui permet d'aller plus loin ${ }^{52}$.» Tout le problème est justement de savoir dans quelle direction va ce «plus loin». Il s'agit, en effet, de savoir si Wolff utilise les mêmes éléments pour produire une définition de la substance qui prenne acte de cette nécessaire médiation par la dynamique de l'action. Or, les quelques éléments que l'on vient d'aborder indiquent plutôt que Wolff utilise l'abstraction pour tenter de faire de la dynamique une science composée d'axiomes: en effet, il indique que, comme la définition féconde des forces est cachée, c'est ce qui justifie qu'il «pose des axiomes» - ce que Jean École désigne comme une «dynamique des principes» dans son introduction à l'Opus metaphysicum ${ }^{53}$.

Dans l'introduction des Principia dynamica, Wolff écrit: «En suivant l'intention de Leibniz dans sa Dynamique, ces axiomata concernent les forces et les actions des corps, indépendamment des phénomènes, en se détournant le plus possible des choses sensibles, nous les dérivons des notions seules $[\ldots]^{54}$.» Il apparaît ici que le mode de démonstration privilégié par Wolff est un mode a priori qui procède selon les raisons des choses. Or, il nous semble, précisément, que cette dimension cachée de la fécondité de la définition des forces est pour Leibniz, en 1692 comme en 1700, une caractéristique essentielle de la dimension heuristique de sa pensée, qui fait sans cesse appel à autre chose: de la force à l'action et de l'action à la perception. Or, cette dimension ouverte du texte leibnizien est interprétée en un tout autre sens par Wolff qui, au contraire, y répond en posant des principes dynamiques comme l'indique le titre de son texte Principia dynamica. De sorte que si Wolff entend l'importance de la métaphysique dans la formulation des principes de la dynamique, c'est au sens où il convoque un certain nombre de principes métaphysiques au fondement de sa démonstration.

La question posée était celle de savoir pourquoi, alors que Wolff a en main la démonstration a priori de la dynamique de l'action et la conscience de son possible impact (il dit explicitement dans les Principia dynamica, qu'elle «permet d'aller plus loin», et Leibniz a montré dans sa correspondance avec Wolff en quoi) - pourquoi, donc, Wolff n'en fait-il pas un instrument de redéfinition de la substance? Le projet fermement ancré dans la pensée de Wolff de maintenir la séparation entre les substances perceptives et les autres permet de comprendre pourquoi la substance de Wolff est physique, puisqu'elle ne débouche pas sur une substance active par soi. On peut dire que, paradoxalement, la diffusion de la dynamique leibnizienne qu'opère Wolff n'est pas déformée, mais c'est la compréhension de son impact effectif pour la métaphysique qui est négligée. Wolff reprend la dimension métaphysique de la dynamique, mais il lui confère une signification qui lui est propre: s'appuyer sur les principes métaphysiques plutôt que de voir au cœur de la dynamique la présence d'une action métaphysique, qui signale l'ambivalence de l'action chez Leibniz. Mais il choisit de ne pas prendre

52. WolfF, 1728, ici 1974, p. 163.

53. ÉCOLE, 1985.

54. WoLfF, 1728, ici 1974, p. 150. 
acte de la dimension dynamique de la métaphysique chez Leibniz, voilà pourquoi sa conception de la substance est celle d'éléments, c'est-à-dire d'unités physiques douées d'une force active physique. On a essayé de montrer ici que ce choix est régi par une divergence constitutive sur le statut de la perception et son incidence sur la délimitation entre les formes de substance. En un mot, si Wolff reprend, tout en la modifiant, la dimension métaphysique du principe de conservation de l'action, il n'en déduit pas une ambivalence de l'action. 


\section{LISTE DES RÉFÉRENCES}

I-Sources

LEIBNIZ (Gottfried Wilhelm), 1689-1690, Dynamica de potentia et legibus naturae corporeae, rééd. dans Mathematische Schriften, vol. VI, éd. Carl I. Gerhardt, Hildesheim/New York, Olms, 1971, p. 281-514.

Leibniz (G.W.), 1694, De la réforme de la philosophie première, dans LeIBNIz, 1978, p. 79-82.

Leibniz (G.W.), 1695, Système nouveau de la nature et de la communication des substances, rééd. dans Système nouveau de la nature et de la communication des substances et autres textes : 1690-1703, éd. Christiane Frémont, Paris, Flammarion, 1994, p. 65-90.

Leibniz (G.W.), 1698, De ipsa natura, dans LeIBNIZ, 1978, p. 93-112.

LEIBNIZ (G.W.), 1699-1701, Essay de dynamique sur les lois du mouvement où il est montré qu'il ne se conserve pas la même quantité de mouvement, mais la même force absolue, ou bien la même quantité d'action motrice, rééd. dans Mathematische Schriften, vol. VI, éd. Carl I. Gerhardt, Hildesheim/New York, Olms, 1971, p. 215-231.

Leibniz (G.W.), 1705-1716, Briefwechsel zwischen Leibniz und Christian Wolff, rééd. Carl I. Gerhardt, Halle, 1860, ici Hildesheim/New York, Olms, 1971.

Leibniz (G.W.), 1978, Opuscules philosophiques choisis, traduits et édités par Paul Schrecker, Paris, Vrin.

Newton (Isaac), 1687, Principes mathématiques de la philosophie naturelle, nouvelle traduction (partielle), postface et bibliographie établies par Marie-Françoise Biarnais, Paris, Christian Bourgois (Épistémè), 1985.

WolfF (Christian), 1728, Principia dynamica, dans Commentarii academiae petropolitanae, t. I (année 1726), rééd. dans Gesammelte Werke, Abt. Lateinische Schriften, II. 35, Hildesheim, Olms, 1974, p. 149-166.

Wolff (C.), 1730, Philosophia prima sive ontologia, rééd. dans Gesammelte Werke, Abt. Lateinische Schriften, II. 3, éd. Jean École, Hildesheim, Olms, 1962.

WolfF (C.), 1731, Cosmologia generalis, methodo scientifica pertractata, qua ad solidam, inprimis dei atque naturae cognitionem via sternitur, Francfort/Leipzig, rééd. dans Gesammelte Werke, Abt. Lateinische Schriften, II. 4, Hildesheim, Olms, 1964.

WolfF (C.), 1734, Psychologia rationalis, methodo scientifica pertractata, Francfort/Leipzig, rééd. dans Gesammelte Werke, Abt. Lateinische Schriften, II. 6, Hildesheim, Olms, 1972.

\section{II - Littérature secondaire}

Belaval (Yvon), 1976, Études leibniziennes. De Leibniz à Hegel, Paris, Gallimard.

Costabel (Pierre), 1981, Leibniz et la dynamique en 1692 : textes et commentaires, Paris, Vrin.

Duchesneau (François), 1994, La Dynamique de Leibniz, Paris, Vrin (Mathesis).

École (Jean), 1964, «Cosmologie wolffienne et dynamique leibnizienne», Les Études philosophiques, $\mathrm{n}^{\circ} 1$, p. 3-9.

École (J.), 1985, Introduction à l'Opus metaphysicum, Paris, Vrin. 
Fichant (Michel), 1998, Science et métaphysique dans Descartes et Leibniz, Paris, Presses universitaires de France.

Fichant (M.), 2004, «L'invention métaphysique», introduction à LeIBNIZ (Gottfried W.), Discours de métaphysique suivi de la Monadologie et autres textes, éd. Michel Fichant, Paris, Gallimard (Folio, Essais), p. 7-140.

JAMMER (Max), 1957, Concepts of force. A study in the foundations of dynamics, Harvard, ici Dover Publications, Mineola, NY, 1999.

Phemister (Pauline), 2005, Leibniz and the natural world. Activity, passivity and corporeal substances in Leibniz's philosophy, Dordrecht, Springer. 